\title{
Solid state AC electroosmosis micro pump on a Chip
}

\author{
Florian L'Hostis ${ }^{1,2}$, Nicolas G Green ${ }^{2}$, Hywel Morgan², Maan Alkaisi ${ }^{3}$ \\ ${ }^{1}$ Electronic and Computer engineering, University of Canterbury, Room A218, Private Bag 4800 New Zealand. \\ e-mail: fah28@student.canterbury.ac.nz,+6433642987 ext 8133 \\ ${ }^{2}$ School of Electronic and computer science, University of Southampton, UK \\ ${ }^{3}$ Electronic and Computer engineering, University of Canterbury, New Zealand
}

\begin{abstract}
Lab-On-a-Chip (LOC) or Micro Total Analysis System ( $\mu$ TAS) technology requires precise control of minute amounts of liquid. Moving liquids in small capillaries requires bulky expensive external pumps that defy the purpose of microfabrication. By integrating a micropump into the device, it allows the system to be transportable, reliable, energy efficient and inexpensive. This system encompasses a solid-state AC electroosmotic pump for the manipulation of liquid containing cells or molecules. This paper reports extensively on the investigation of the pumping ability of the solid state array with KCl solution. Fluorescent beads of $500 \mathrm{~nm}$ where used as tracer to monitor the fluid velocity. Two different array geometries were investigated as well as different material for the electrodes. Dielectorphoretic trapping of nanoparticles and cells was achieved. This paper will discuss the inherent problem of an AC electroosmosis driven micropump for lab on chip applications as well as the fluid flow yield in both directions and magnitude in correlation with electric field intensity and frequency.
\end{abstract}

Keywords- AC electroosmosis, micropump, microfluidic ,nano particle trap, Dielectrophoresis, Cell trap.

\section{INTRODUCTION}

Microfabricated devices have generated a lot of interest across the globe. LOC or its other embodiment $\mu$ TAS offer new paradigms in the design and applications for the life science industry but also the wide range of pharmaceutical to chemical industrial applications. In these systems all the function necessary to operate the device are integrated into the same substrate. Therefore the need for control of minute amount of liquid is paramount. This control is under the umbrella of Microfluidics [1] encompassing several areas of science. Many methods are known to control liquid in LOC such as micromechanical, electroosmosis and electrowetting. Dynamic micropumps based on electrohydrodynamic and magnetohydrodynamic effects have also been developed [2]. Two distinction are made among these devices : with or without moving part. Although Mechanicals micropumps have shown success the downscaling factor is limited and they are also more susceptible to jamming and breaking than the no moving part micropumps. Nevertheless two regimes are available in electrokinetic micropumps $\mathrm{AC}$ and DC. The latest requires high voltage and therefore is not suitable for the study of living cell. Furthermore electrolysis occurs and creates bubbles. AC electroosmosis has recently been observed to create fluid motion within a certain domain of frequency and media conductivity over microfabricated electrodes [3]. First predicted by Ajdari [4], the pumping ability of AC electroosmosis was soon after demonstrated by Brown et al. [5]. AC Electroosmosis is the movement of induced charges over polarised electrodes created by a non-uniform electric field. The tangential component of the field over the electrode accelerates charges and drags the fluid with their motion. This results in bulk flow over the electrodes. The fluid motion has a steady state that is reached almost immediately upon polarization of the electrodes. Experiments have been reported by many groups [6-11] with diverse degree of success. The first experimental drawback proved the inability to create backpressure. It lead Studer et al [8] and Debesset [11] et al to design a close loop channel to study the pumping ability on one hand and a chromatographic device on the second hand. Since the micropump lays on the substrate and the micro channel are constructed above it is natural to think of improving the pumping ability by using the same pumping system as the lid to close the channel. Mpholo et al [7] investigated such a device and the plug flow resulting on both side of the pump (Top and Bottom configuration) was studied. Nevertheless its investigation remains in the linear study boundaries of Ramos et al [12] and they did not observe the reversal flow [8]. Olesen et Al [13] recently extended the existing theory and investigated Faradaic currents as a source of the observed reversal as well as the effect of geometrical confinement such as Studer et al case [8].

The necessity of transportable LOC raises an iterest for such AC electroomotic devices. Based on previous research it is posssible to design a closed loop system for dielectrophoretic chromatography [14] - it is a demonstrator that is well suited with the electrical field nature of $\mathrm{AC}$ electrosomosis. In order to increase the pumping a top and bottom geometry is investigated [7], which we call double sided pump and a single sided pump was designed. This is described with the rest of the experimental protocol in section II. We will present the result of this approach in section III and conclude in section IV.

\section{DEVICE GEOMETRY AND DESIGN}

\section{A. Microfluidic system design.}

The microfluidic chip is designed to produce a steady flow in a central channel where a Dielectrophoresis trap (DEP) is microfabricated using the same material as the AC electroosomotic micropump in the same lithographic step Figure 1 and 2. DEP is defined as the lateral motion imparted on uncharged particles as a result of polarization induced by 
non-uniform electric fields [15]. The trap is made of two components. Cells can be studied more carefully in their dielectric response and the spherical layered particle model develop by Jones [16]

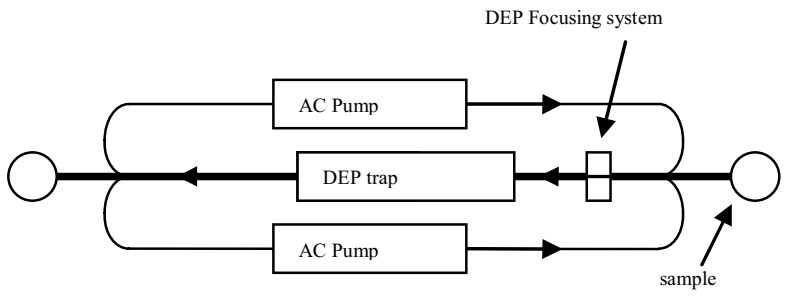

Figure 1: Device Schematic

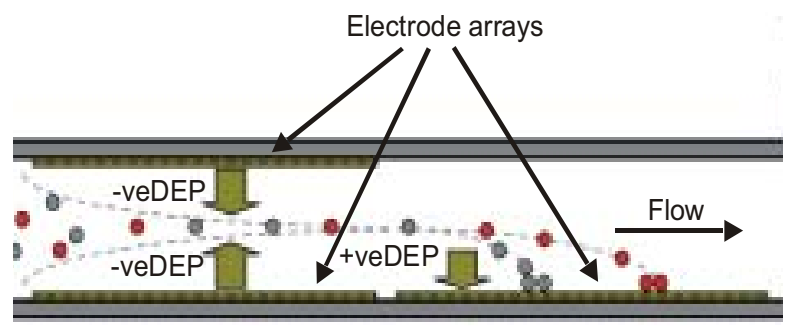

Figure 2 : Microfabricated double sided device

Two systems are built in order to evaluate the pumping. One side of the pump is used with a spacer where a transparent lid is placed on top. The spacer is either a double layer of 75 micron of ordyl dry film resist or a double sided tape of 150 micron thickness. Access ports are drilled into the superior lid or the liquid media is inserted via capillary action. The second system is built the same way and in case of the dry film resist the bonding is achieved with the help of thermal treatment cyles with an applied pressure. The system remain open ended to balance pressures and avoid disturbing the fluid flow within the pump loop

\section{B. Electrodes design}

The DEP focusing system operated at higher frequency to repel particle at the center of the channel where they experience the strongest and identical fluid drag force. The Trap is made of regularly spaced electrodes with a gap and electrode width of 40 micron. The dielectrophoretic focusing system is made of interdigitated electrode of first 20 micron then 40 micron wide electrodes[14]. DEP forces are short distance force that extend to about the size of the pitch. The magnitude of the force is increased with the non uniformity of the field. Hence the smaller the electrode the more diverging the field and the stronger the force. The first series of electrodes brings the particle closer to the centre of the channel and the last series focuses them in the same fluid velocity sheets..
Both the DEP trap and the ACEO micropump are made of either a Ti/Au of $20 / 160 \mathrm{~nm}$ respectively or a sandwich of $\mathrm{Ti} / \mathrm{Au} / \mathrm{Ti}$ of $20 / 160 / 20 \mathrm{~nm}$. We propose to investigate which metal would be more appropriate since Ti grow a natural oxide. $\mathrm{Ti}$ is more resistive but less susceptible in generating faradaic current. The Electrosmotic pump is made of an 800 repeat of a base unit. This base unit is based on structure proposed by [12] where: $\mathrm{W} 1=\mathrm{G} 1=5$ microns, $\mathrm{W} 2=\mathrm{G} 2=13$ microns.

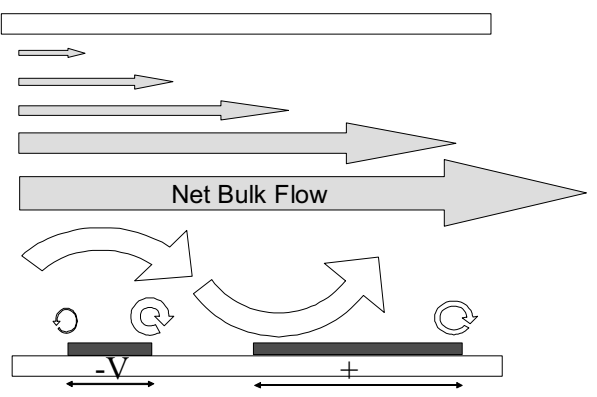

Figure 3 : One side ACEO micropump

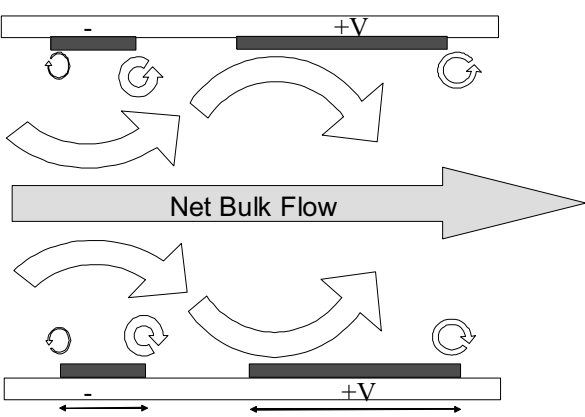

Figure 4: Double sided ACEO micropump

The first system was used for prototyping the second.. We observe from figure 3 the non slip boundary condition on the lid whereas figure 4 as a symmetry and will be less affected by recirculation and then create a plug flow as already demonstrated by Mpholo [7].

\section{Measurement protocols}

The system is intended to create a minimum of $40 \mathrm{micron} / \mathrm{s}$ fluid velocity. Since the system creating a flow on its surface we can neglect the effect of the wall of the channel. The channel are $3-5 \mathrm{~mm}$ wide and 150 micron height. The velocity are observed by video recording using a tailored microscope system. The $500 \mathrm{~nm}$ bead pass in front of a $* 10$ or $* 20$ nikon objective as demonstrated in Figure 5. The velocity measurement is nearly impossible for the double sided electrode since it is very difficult to observe the tracer beads through the electrode unless it is made of ITO transparent material[7]. 


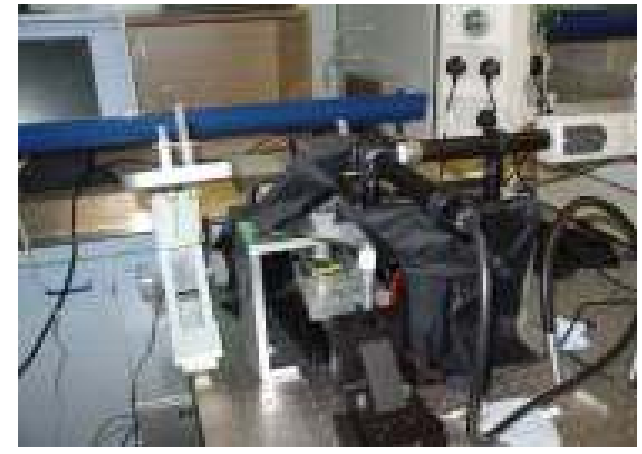

Figure 5 : Microscope use to investigate fluid flow

The velocity measurements are always observed at the middle of the central channel at mid height. Since the beads have a tendency to adhere to the electrode we always re-inject new media with beads after each measurement. Although we observed little change on the velocity for the same experimental conditions with or without the bead collected at the ACEO electrode. We measured the conductivity on the day of the experiment and the addition of beads did not modify the bulk conductivity significantly even for low concentration of $\mathrm{KCl}$.

In order to observe the velocity we overlay 25 consecutive frame over the same image. The image then shows the distant covered in $1 \mathrm{sec}$ by a bead. Only the bead in focus are used and as many as possible are used to measure the distance cross over a second as shown in Figure 6

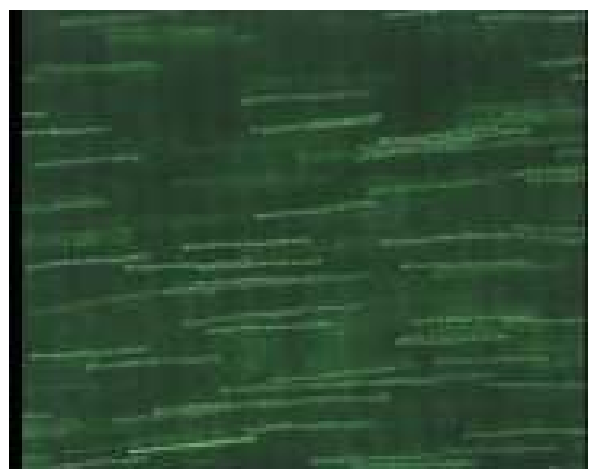

Figure 6 : Fluid velocity measurement using tracer beads

We used an alpha impedance analyzer to observe the formation of the double layer and its relaxation. The device is monitored by the analyzer and we sweep in frequency from 10 $\mathrm{Hz}$ to $1 \mathrm{MHz}$. For each frequency cycle we increase the voltage to a maximum of $3 \mathrm{Vrms}$ available by the system .

\section{RESULTS AND DISCUSSION}

The first important study was to observe fluid flow and to characterize the most efficient material for Integrated ACEO micropump. The study with the Impedance analyzer showed for gold a constant decrease of the phase angle for low frequency. This means that the electric double layer was slowly vanishing and the system was becoming resistive with a very low resistance. This can only conclude to a short circuit created between a certain number of electrode. Nevertheless the impedance before exposing the electrode to voltage with a $\mathrm{KCl}$ solution show a very high resistance in DC. This disappears after a series of cycles at $3 \mathrm{Vrms}$ from $10 \mathrm{~Hz}$ to 1 $\mathrm{MHz}$.

\section{1) Material effect on ACEO micropump performance}

We assembled several one sided system of $\mathrm{Ti} / \mathrm{Au}$ and observed the degradation of pumping to reach almost complete stop. The samples were observed with a scanning electron microscope and it is obvious that a growth of conducting material happens between the electrodes as shown on figure 7 .

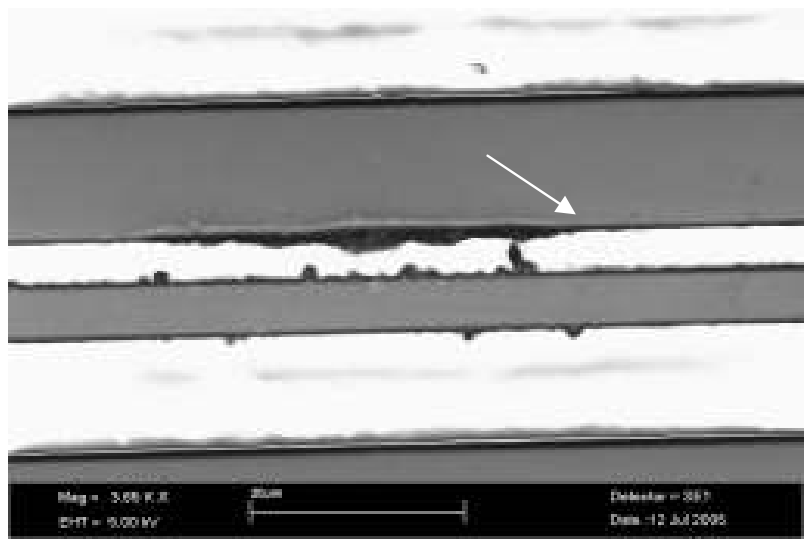

Figure 7: Gold microelectrode short circuited as indicated by the arrow

The electrode where the gold is encapsulated show no such degradation but we observe some discrepancy on the electric double layer EDL formation with the impedance analyzer. We observe as well transient effect for a continuous voltage. The experiment was performed at $1000 \mathrm{~Hz}$ and $10 \mathrm{Vpp}$. After a normal flow direction expected by the linear theory [12] chaotic flow is observed before settling in the reversal. If the device cleaned and polarized in the same way reversal immediately occurs. This passivates the Ti layer occurring at the surface of the electrode adding a capacitance in series with the EDL and faradaic current that change with the formation of the passive layer. This observation does not attribute to the reversal to only faradaic current but highlights the change of behavior for different materials. We have noted that Gold Chloride is likely to be responsible for the dissolution and its re-deposition might lead to short circuit the electrodes.

\section{2) Velocity Measurement for double sided pump}

We observed reversal for both $1.5 \mathrm{mS} / \mathrm{m}$ and $10 \mathrm{mS} / \mathrm{m}$ at diverse range of frequencies and applied voltages. Nature of this system and its mutliparameter make it hard to understand so we draw both curve into a contour to establish a mapping of velocities on figure 8 and 9. (note that some intermediate contour were removed for ease of reading). 


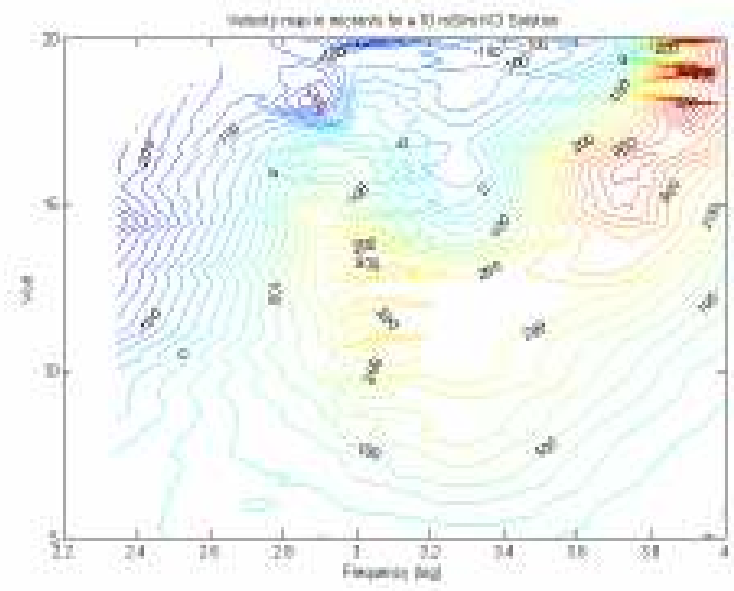

Figure 8: Velocity map for a $10 \mathrm{mS} / \mathrm{m} \mathrm{KCl} \mathrm{Solution}$

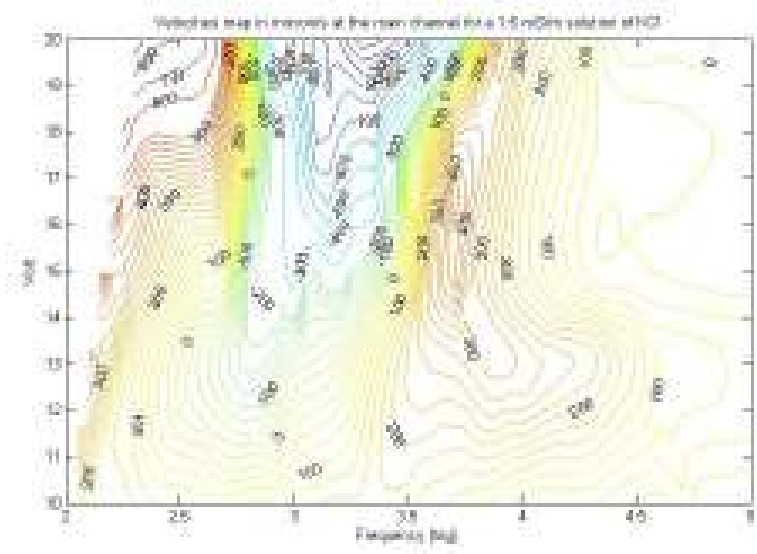

Figure 9: Velocity map for a $1 \mathrm{mS} / \mathrm{m} \mathrm{KCl}$ Solution

Both mappings show reversal around $1 \mathrm{kHz}$ to $5 \mathrm{kHz}$ with a maximum velocity occurring in reversal. The reversal is stronger and more defined for low saline concentration which is in agreement with Studer [8] who attributed the increase of velocity in reversal on lower saline concentration. Nevertheless in the case Studer system it occurs at higher frequency by a factor 10. Our system presents the least geometrical confinement because of the double sided micro pump. Studer geometry [8] is confined and the material used for the electrode is Platinum. From figure 8 we observe a low voltage quadratic trend but nothing similar to the $1 \mathrm{mS} / \mathrm{m}$ case. We note similar results in reversal flow but not in the magnitude observed by Garcia etal for traveling wave electokinetic micropumping when using Titanium for electrodes [9]. The magnitude is certainly different since 4 pumps are actively displacing water into the main channel. This prove the existence of reversal flow in the top and bottom electrode for creating plug flow in closed loop.

Although several groups have proposed the hypothesis of a reversal driven by Faradaic current it seems from what we observed that other parameter influence the reversal flow such as the intrinsic ACEO design, material used for the electrode and the ionic content of the media used. The reversal occurrence at low conductivity raises the question of local salt depletion as presented by [13]. Other theoretical arguments can be investigated and a more thorough investigation will be published elsewhere

\section{CONCLUSION}

Investigation of microfluidic pumping was achieved in both "reversal and normal" flow direction at different time frequency and liquid conductivity. This device successfully integrates several techniques for cells and nanoparticle sorting and chracterisation and has applications in biomedical and rapid diagnostic.

\section{ACKNOWLEDGMENT}

The Authors wish to acknowledge the contribution of the Optelectronic Research Centre and INNOS for fabricating the device. Florian L'Hostis would like to thank the MacDiarmid institute. This work was helped by many interesting topic discussion with Pablo Garcia, Dr A. Ramos, Prof T.B. Jones.

\section{REFERENCES}

[1] "Engineering flows in small devices: microfluidics towards a labon-a-chip" H.A. Stone, A.D. Stroock, A. Ajdari Annual Review of Fluid Mechanics, January 2004, Vol. 36, Pages 381-411

[2] "A Review" D.J. Laser and J.G. Santiago, Journal of Micromechanics and Microengineering, vol 14, no. 6, p. R35-R64, 2004

[3] "fluid flow induced by nonuniform ac electric fields in electrolytes on microelectrodes a linear double-layer analysis". N. G. Green, H. Morgan, A. Ramos et al Phys. Rev. April 2000. Volume 61, Number 4.

[4] "Pumping liquids using asymmetric electrode arrays" A. Ajdari, Phys Rev E 61 R45 (2000).

[5] "Pumping of water with ac electric fields applied to asymmetric pairs of microelectrodes " A. B. D. Brown, C. G. Smith, A. R. Rennie, Phys. Rev.E 63, 016305 (2000).

[6] "Fabrication of microfluidic devices for AC electrokinetic fluid pumpingl" V. Studer, A. Pepin, Y. Chen, A. Ajdari, Microelectron. Eng. 61-62, 915 (2002)

[7] "Low voltage plug flow pumping using anisotropic electrode arrays "M. Mpholo, C. G. Smith, A. B. D. Brown, Sens. Actuators B 92, 262 (2003)

[8] "An integrated AC electrokinetic pump in a microfluidic loop for fast and tunable flow control'V. Studer, A. Pepin, Y. Chen, A. Ajdari, The Analyst 129, 944 (2004)

[9] P. Garcia, A. Ramos, N. G. Green, H. Morgan, Proc.IEEE Int. Conf. Dielectric Liquids 2005, 181-184.

[10] "Electrokinetic micropump and micromixer design based on ac faradaic polarization,"D. Lastochkin, R. Zhou, P. Wang, Y. Ben, H.-C. Chang,J. Appl. Phys. 96, 1730 (2004).

[11] "An AC electroosmotic micropump for circular chromatographic applications" S. Debesset, C. J. Hayden, C. Dalton, J. C. T. Eijkel and A. Manz Lab on a Chip, 2004, 4, $396-400$

[12] "Pumping of liquids with ac voltages applied to asymmetric pairs of microelectrodes" A. Ramos, A.González, A. Castellanos, N. G. Green, and H. Morgan Phys. Rev. E 67, 056302 (2003).

[13] "AC electrokinetic micropumps: the effect of geometrical confinement, Faradaic current injection, and nonlinear surface capacitance" L. Olesen, H. Bruus, A. Ajdari, in press, 2006.

[14] ] "Microdevices for Dielectrophoretic Flow through Cell separation" D. Holmes. N. G. Green. H. Morgan. IEEE Engineering in Medicine and Biology.Vol 2. Nb 6 Nov/Dec 2003.

[15] "Dielectrophoresis: The Behavior of Neutral Matter in Nonuniform Electric Fields", Pohl, H. A., Cambridge University Press, Cambridge 1978, pp. 1-579.

[16] T. B. Jones. "Electromechanics of Particles", Cambridge University Press, New York City, NY, 1995. 